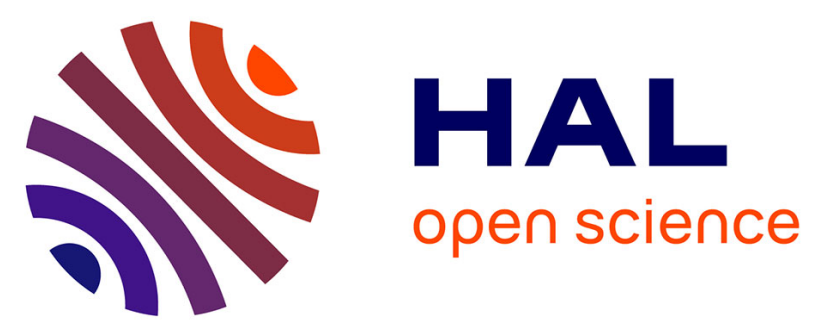

\title{
Phénomènes oscillatoires à l'interface acier inoxydable 3161 - albumine humaine en milieu biologique simulé
}

\author{
M. El Ouahabi, F. El Mdari, M. Marie de Ficquelmont-Loïzos, Hisasi
}

Takenouti, P. Lambin, S. El Adioui, A. Srhiri

\section{- To cite this version:}

M. El Ouahabi, F. El Mdari, M. Marie de Ficquelmont-Loïzos, Hisasi Takenouti, P. Lambin, et al.. Phénomènes oscillatoires à l'interface acier inoxydable $316 \mathrm{l}$ - albumine humaine en milieu biologique simulé. Matériaux \& Techniques, 2002, 90 (7-8), pp.49 - 54. 10.1051/mattech/200290070049 . hal01783555

\section{HAL Id: hal-01783555 \\ https://hal.sorbonne-universite.fr/hal-01783555}

Submitted on 2 May 2018

HAL is a multi-disciplinary open access archive for the deposit and dissemination of scientific research documents, whether they are published or not. The documents may come from teaching and research institutions in France or abroad, or from public or private research centers.
L'archive ouverte pluridisciplinaire HAL, est destinée au dépôt et à la diffusion de documents scientifiques de niveau recherche, publiés ou non, émanant des établissements d'enseignement et de recherche français ou étrangers, des laboratoires publics ou privés. 


\title{
PHÉNOMÈNES OSCILLATOIRES À L'INTERFACE ACIER INOXYDABLE 316L - ALBUMINE HUMAINE EN MILIEU BIOLOGIQUE SIMULÉ
}

\author{
M. El Ouahabi', F. El Mdari², M. Marie de Ficquelmont-Loïzos', \\ H. Takenouti ${ }^{3}$, P. Lambin ${ }^{4}$, S. El Adioui ${ }^{2}$ et A. Srhiri ${ }^{5}$ \\ 1) LBHP ESA 7057 CNRS, Fédération de Recherche FR 2438 \\ "Matière et Systèmes Complexes", Université Denis-Diderot, Paris \\ 2) Laboratoire de Biophysique, Faculté de Médecine, \\ Université Hassan II - Ain Chock, Casablansa, Maroc \\ 3) UPR 15 CNRS, Université P. et M. Curie, Paris \\ 4) INTS, Paris \\ 5) LEECE, Faculté des Sciences, Université Ibn Tofaïl, Kénitra, Maroć
}

\section{OSCILLATING PHENOMENA AT THE 3I6L STAINLESS STEEL-HUMAN ALBUMIN INTERFACE IN SIMULATED BIOLOGICAL MEDIUM}

ABSTRACT: The behaviour of a mating disc electrode construcled in an atstentitic $3 / 6 \mathrm{~L}$ stain. less steel, at the corrosion potential, was studied, through electrochemical impedante spertmscopy, in wo aqueous media partly simulating biological media, namely: $0.1 \mathrm{M}$ phosphate buffer $+9 g / L ~ N a C l(p H=7.4)$ and $0.1 \mathrm{M}$ phosphate buffer $+9 g / L . ~ N a C l+1 g / L$ human serum albumin $(\mathrm{pH}=7.4)$ at $37^{\circ} \mathrm{C}$ and ander permanent laminar flow (rotation speced of $700 \mathrm{rmm}$. hence shear rate of $1000 \mathrm{~s}^{-1}$ ). Two types of measurements have been performed, i.e. at a given time and as a function of time. For both media and both types of measurements, the Nyeruist diagrams are fitted by wo depressed loops, corresponding to an electrical equivalent circuit imolving two parallei $R-C$ circuits with two $C P E$. The high frequency loop was attributed to the double layer and space charge, and the low frequency loop to the formation of a passive film at the electrode surface. At a given time, just after electrode immersion, the interface electrical parameters indicate that albumin inhibits pitting corrosion. Contrarily, measurements as a function of time show that albumin affects all the parameters relative to the double layer (but one) and to the film, and that they exhibit oscillations with time at different degrees. The behaviour of the charge transfer resistance and that of the dispersion coefficient of the film faradaie capacitance, show that the protein inhibits or favours pinting corrosion in an alternating manner: This oscillating phenomenon was interpreted in terms of an adsorption-desorption serpuence of the protein under the influence of shear:

KEY WORDS: Adsorption-desorption; Dowble laver capacitance: Electrochemical Impedance Spectroscopy; Bome Implants; C.P.E.. Shear Flow:

\footnotetext{
Conférence présentée au Forum "Biodétérioration des matéricux" organisé les 23 et 24 mai 2002 à Dourdan par la Commission Biodétérioration des Matériaux du Centre français de l'Anticorrosion (Cefracor) avec le parrainage de la Société française de Microbiologie.
}

RÉSUMÉ: Le comportement d'une électrode à disque tournant en acier inoxydable austénilique $316 \mathrm{~L}$, au potentiel de corrosion, a été étudié, par spectroscopie d’impédance électrochimique. dans deux milieux simulant partiellement des milieux biologiques: tampon phosphate $0.1 \mathrm{M}+$ $\mathrm{NaCl} 9 \mathrm{~g} / \mathrm{L}(\mathrm{pH}=7,4)$ et tampon phosphate $0,1 \mathrm{M}+\mathrm{NaCl} 9 \mathrm{~g} / \mathrm{L}$ + sérum albumine humaine a $1 \mathrm{~g} / \mathrm{L}(\mathrm{pH}=7,4)$ à $37^{\circ} \mathrm{C}$ et sous écoulement laminaire permanent (vitesse de rotation de

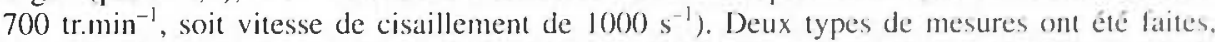
à un temps donné et en fonction du temps. Pour les deux milieux et pour les deux ypes de mesure, les diagrammes de Nyquist sont ajustés par deux boucles affaissées. qui correspondent à un circuit électrique équivalent comportant deux circuits $\mathrm{RC}$ en parallè le avec deux CPE. L.a boucle en hautes fréquences est attribuée à la double couche et à la charge d'espace. et la boucle en basses fréquences à la formation d’un film passif à la surface de l'électrode. A un temps donné, dès le contact acier-milieu, les parametres électriques de l’interface indiquent que 
l'albumine inhibe la corrosion par piqûres. Par contre, les mesures en fonction du temps révèlent que l'albumine affecte tous les paramètres relatifís à la double couche (siluf un) et au film, et que ceux-ci présentent, à des degrés différents, des oscillations en fonction du temps. Le comportement de la résistance de transfert de charge et celui du coefficient de dispersion de la capacité faradique du film montrent que la protéine inhibe ou favorise alternativement la corrosion par piqûres. Ce phénomène oscillatoire a élé interprété par une séquence adsorption-désorption de la protéine sous l'effet de l'écoulement.

Mơs clefF: Adsorption - désorption; Capacité de double couche; Spectroscopie d'Impédance Electrochimique; Implants osseux ; C.P.E.; Ecoulement de cisaillement.

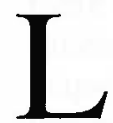
es aciers inoxydables représentent une grande majorité des biomatériaux métalliques utilisés en chirurgie orthopédique et dentaire. Ils se trouvent toujours au contact de fluides biologiques qui sont très agressifs du fait de la présence, entre autres, d'ions chlorure, espèce la plus agressive existant dans ces milieux, et des macrophages, secrétant des substances extrêmement acides, sans parler de la formation éventuelle d'un biofilm causant des infections bactériennes [1,2]. Ce contact conduit à une corrosion localisée, qui peut causer une rupture de l'implant, et à des réactions inflammatoires, des nécroses ou autres, suite aux produits de corrosion. Ces échecs posent encore de nombreux problèmes à la fois sur le plan clinique et fondamental, mais aussi socio-économique. L'étude du comportement du système acier-fluide biologique est donc d'une grande importance, mais ces problèmes sont d'autant plus complexes que les résultats sur la corrosion obtenus in vitro dans des milieux simulant des fluides biologiques diffèrent souvent des résultats in vivo [3-9].

Ces différences pourraient être dues à l'adsorption, inévitable, de protéines présentes dans les fluides biologiques $[10,11]$. Une autre difficulté vient du fait que chaque couple protéinesubstrat est un cas en soi dont le comportement, en ce qui concerne la corrosion, dépend des conditions expérimentales, ce qui explique les contradictions apparentes reportées dans la littérature $[3,4,7,8,12-15]$. Ainsi, la corrosion par frottement ou par piqûres d'aciers inoxydables (dont l'acier 316L) dépend de la nature et de la charge de la protéine, donc du pH de la solution, du potentiel, etc. [16].

Mais aucune de ces études, du moins à notre connaissance, ne considère l'évolution du comportement de l'interface au cours du temps. Or, il est bien connu que le devenir d'un implant est fortement lié au paramètre temps, des échecs pouvant survenir à plus ou moins longue échéance.

Nous présentons ici une étude concernant l'influence du temps sur la corrosion de l'acier inoxydable 316L utilisé en ostéosynthèse. en présence d'une protéine plasmatique humaine, la sérum albumine, dans des conditions de température et de $\mathrm{pH}$ proches des conditions physiologiques. La biocompatibilité d'un matériau pouvant être affectée par les contraintes auxquelles il est soumis, l'étude a été menée sous contrainte de cisaillement.

Du fait de la présence simultanée de deux phénomènes aux interfaces hétérogènes. l'accumulation des protéines et la présence de groupes chargés, une méthode électrochimique a été mise en couvre ici, celle de la spectroscopie d'impédance électrochimique (SIE) au moyen de l'électrode à disque tournant. Cette méthode, largement utilisée dans le cas de la corrosion, peut aussi fournir des informations sur l'adsorption des protéines $[17,18]$.

\section{Matériels et méthodes}

Deux milieux aqueux (eau ultra-pure, appareil Elga Stat UHQII. Millipore) ont été étudiés: une solution de $\mathrm{NaCl}$ à $9 \mathrm{~g} / \mathrm{L}$ contenant du tampon phosphate $0,1 \mathrm{M}$ à pH $=7,4$ (nommé ici TAMP) et une solution de sérum albumine humaine (HSA) i $1 \mathrm{~g} / \mathrm{L}$ dans le milieu TAMP, également à $\mathrm{pH}=7,4$.

Les mesures ont été effectuées à une température de $37 \pm 0,1^{\circ} \mathrm{r}$ (cryostat Ministat Numérique MHUB 11) sous régulation potell tiostatique avec une électrode à disque tournant (EDT), un électrode de référence (calomel saturé, ECS) et une contre électrode (grille de platine hémisphérique).

L'EDT étudiée est préparée à partir d'une vis en acier inoxy dable $316 \mathrm{~L}$ (nommé ici $\mathrm{AO}$ ) utilisée pour le serrage de plaque d'ostéosynthèse (diamètre de la partie active $=4,67 \mathrm{~mm}$ ). $\mathrm{L}$ composition de cet alliage est : $0,013 \% \mathrm{C} ; 19,4 \% \mathrm{Cr} ; 14,5 \% \mathrm{Ni}$ $2,6 \% \mathrm{Mo} ; 0,7 \% \mathrm{Si}$; et fer jusqu'à $100 \%$. La partie latérale d cylindre est enrobée d'une résine époxy inerte et non conductr ce. Cette électrode a été polie (poli miroir) avant chaque ess: avec un papier abrasif (granulométrie 600) et par une susper sion d'alumine de granulométrie décroissante $(9$ et $1 \mu \mathrm{m}$ mone dispersée), puis passée deux fois aux ultrasons.

Tous les essais ont été effectués dans des conditions hydrod: namiques bien déterminées (régime laminaire permanent), une vitesse de rotation de $700 \mathrm{tr} \cdot \mathrm{min}^{-1}$; ce qui correspon dans le cas de l'écoulement généré par l'EDT, à une vitesse d cisaillement $\dot{\gamma}$ de $1000 \mathrm{~s}^{-1}$.

Le comportement de l'interface a été étudié en fonction d temps par spectroscopie d'impédance électrochimique (SIE Cependant, plusieurs autres méthodes (résultats non reporté ici) ont dû être mises en œuvre, préalablement à la SIE, en vu de la détermination de certaines conditions expérimentale: comme la chronopotentiométrie (potentiel de corrosion libre la voltamétrie cyclique (potentiel de piqûration) ou bien I méthode de Mott-Schottky (caractère semi-conducteur du filı passif formé à la surface de l'acier).

La chaine de mesures électrochimiques comprend un potentic stat (1286 Electrochemical Interface, Solartron), un analyser de réponse en fréquence (HF Frequency Response Analyse: modèle 1255, Solartron) et un multimètre numérique (Hewletı Packard, HP 3478 A), pilotés par ordinateur.

La saisie et le traitement des données ont été décrits dans un publication antérieure [17].

\section{Résultats et discussion}

La présente étude, basée sur la spectroscopie d'impédance, si place à la fois sous l'angle de l'influence du temps et celui dı la corrosion. Par conséquent, l'influence de l'albumine su l'interface acier 316L - TAMP a été considérée ici sous deu: aspects: le comportement de l'interface à un instant donné e en fonction du temps, pour les deux milieux (donc en absenc et en présence de protéine).

L'étude par SIE (domaine de fréquence entre $10 \mathrm{kHz}$ et $100 \mathrm{MH}$ : à raison de 5 points par décade) a été faite au potentiel de cor rosion tel qu'il a été déterminé par ailleurs, à savoir la valeur asymptotique de la courbe chronopotentiométrique pour chacu ne des deux solutions $(-140 \mathrm{mV} / \mathrm{ECS}$ pour TAMP et de $-18 \mathrm{C}$ $\mathrm{mV} / \mathrm{ECS}$ pour HSA). 


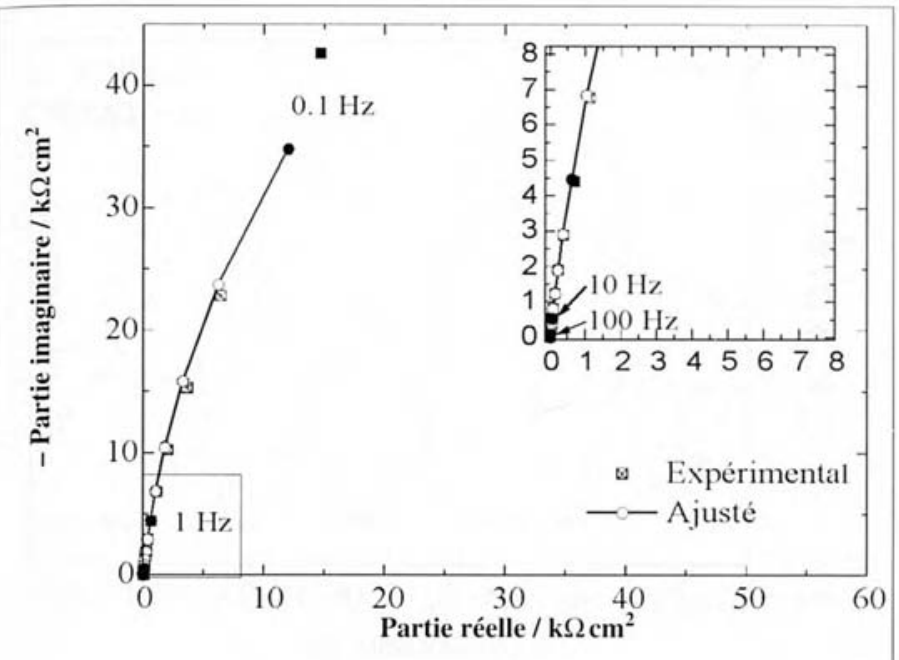

ig. 1 - Ajustement du spectre d'impédance de AO dans TAMP à $t \cong 0$ TAMP $=$ solution aqueuse $\mathrm{NaCl} 9 \mathrm{~g} / \mathrm{L}+\operatorname{tampon}$ phosphate $0,1 \mathrm{M}$ à $\mathrm{pH}=$ ,4); Potentiel = $140 \mathrm{mV} / \mathrm{ECS}$; Vitesse de rotation $=700 \mathrm{tr}^{\mathrm{min}} \mathrm{m}^{-1}$; lempérature $=37^{\circ} \mathrm{C}$.

7ig. 1 - Finting of the impedance spectrum of $A O$ in TAMP at $1 \equiv 0(T A M P=$ cqueous solution of $9 \mathrm{~g} / \mathrm{L}+0.1 \mathrm{M}$ phosphate buffer $a \mathrm{pH}=7.4)$. Potential $=$ $40 \mathrm{mV} / \mathrm{SCE} ;$ Rotation speed $=700 \mathrm{rpm} ;$ Temperature $=37^{\circ} \mathrm{C}$.

\section{Comportement de l'interface à un temps donné}

-es diagrammes de Nyquist (figures 1 et 2) ont été saisis au otentiel choisi et à la vitesse de rotation $\Omega=700 \mathrm{tr}$. $\mathrm{min}^{-1}$, dès 'immersion de l'EDT dans le milieu ( $\mathrm{t} \cong 0$ ), contrairement à les travaux antérieurs où un temps d'immersion de l'EDT l'une heure en circuit ouvert avait été utilisé [19].

J'après une analyse par ajustement de paramètres, il s'est ivéré que pour les deux milieux, il faut introduire deux zonstantes de temps $\left(\tau_{1}, \tau_{2}\right)$, correspondant à un circuit équivaent comportant deux circuits $\mathrm{RC}$ en parallèle (fig. 3), en accord ıvec des résultats de la littérature [20]. En effet, avec un seul zouple $\mathrm{R}$ et $\mathrm{C}$, un écart systématique et important entre points zxpérimentaux et courbe ajustée est observé, ce qui montre une nadéquation du circuit équivalent envisagé. Les deux boucles Iu diagramme étant affaissées, les capacités sont dispersées Constant Phase Element).

Pour les deux milieux, la boucle en hautes fréquences peut être ittribuée à la double couche et à la charge d'espace [19] et la soucle en basses fréquences à la formation d'un film passif à la iurface de l'EDT. Ce film présente un caractère semi-conduceur (droite dans le plan de Mott-Schottky) comme dans le cas, slairement montré, de $\mathrm{NaCl}$ seul [19].

Le tableau I rassemble les grandeurs électriques de l'interface relatives au milieu $\left(R_{e}\right.$, résistance de l'électrolyte), à la double couche $\left(R_{t}\right.$, résistance de transfert de charge; $C_{d}$ capacité de la double couche; $\alpha_{d}$, coefficient de dispersion de $C_{d}$ ) et au film

Tableau I - Paramètres électriques de l'interface acier 316 L - milieu à une date fixée proche de I'immersion de l'EDT $(t=0)$.

Table I - Electrical parameters of the $3 / 6 L$ steel-medium interface at a given time close to EDT immersion $(t \equiv 0)$.

\begin{tabular}{|l|c|c|c|c|c|c|c|}
\hline \multirow{2}{*}{ Milieu } & Électrolyte & \multicolumn{3}{|c|}{ Double couche } & \multicolumn{3}{c|}{ Film } \\
\cline { 2 - 9 } & $\begin{array}{c}\boldsymbol{R}_{e} \\
\Omega \mathrm{cm}^{2}\end{array}$ & $\begin{array}{c}\boldsymbol{R}_{\boldsymbol{t}} \\
\mathrm{k} \Omega \mathrm{cm}^{2}\end{array}$ & $\begin{array}{c}\boldsymbol{C}_{\boldsymbol{d}} \\
\mu \mathrm{F} \mathrm{cm}\end{array}$ & $\alpha_{d}$ & $\begin{array}{c}\boldsymbol{R}_{\boldsymbol{F}} \\
\mathrm{M} \Omega \mathrm{cm}^{2}\end{array}$ & $\begin{array}{c}\boldsymbol{C}_{\boldsymbol{F}} \\
\mu \mathrm{F} \mathrm{cm}\end{array}$ & $\alpha_{\boldsymbol{F}}$ \\
\hline TAMP & 6,59 & 3,84 & 34,28 & 0,838 & 7,42 & 6,83 & 1,00 \\
\hline HSA & 6,81 & 8,31 & 41,69 & 0,924 & 0,072 & 39,92 & 1,00 \\
\hline
\end{tabular}

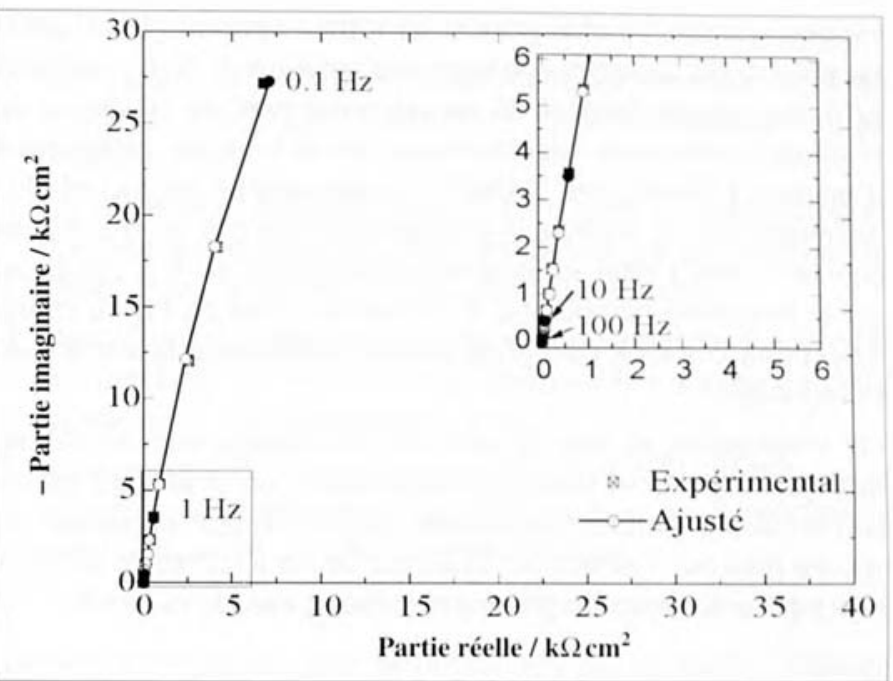

Fig. 2 - Ajustement du spectre d’impédance de AO dans HSA à $t \equiv 0$ (HSA = solution de sérum albumine humaine à I g/L, dans TAMP): Potentiel = $180 \mathrm{mV} / \mathrm{ECS}$; Vitesse de rotation = 700 $\mathrm{tr}_{\mathrm{min}}^{-1}$; Température $=37^{\circ} \mathrm{C}$.

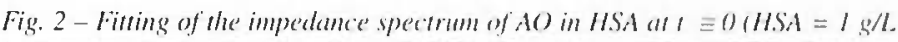

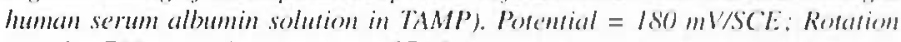
speed $=700 \mathrm{rpm}:$ Temperature $=37^{\circ} \mathrm{C}$.

Fig. 3 - Circuit électrique équivalent adopté pour les deux milieux (TAMP et IISA) $\left(R_{e}\right.$, résistance de l'électrolyte $; R_{t}$, résistance de transfert de charge: $C_{d}$, capacité de la double couche; $\alpha_{d}$, coefficient de dispersion de $\mathrm{C}_{\mathrm{d}}$; $\mathrm{R}_{\mathrm{F}}$, résistance faradique ; $\mathrm{C}_{\mathrm{F}}$, capacité faradique; $\sigma_{\mathrm{F}}$, coeff. de dispersion de $\mathrm{C}_{\mathbf{F}}$ ).

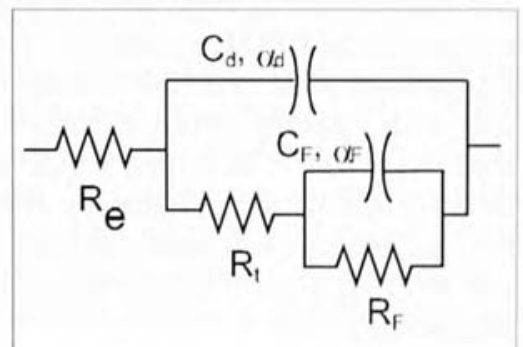

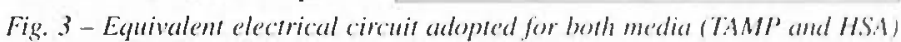

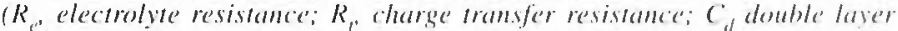
capacinance; $\alpha_{d}$, dispersion coefficient of $C_{d}: R_{f}$, faradaic ressisance; $C_{f}$. faradaic capaciance; $\alpha_{p}$, dispersion coselficiont of $(\%)$.

$\left(R_{F}\right.$, résistance faradique: $C_{F}$, capacité faradique; $\alpha_{F}$, coefficient de dispersion de $C_{F}$ ).

À un temps fixé $(t \cong 0)$, l'influence de la protéine sur les paralmètres électriques de l'interface acier $316 \mathrm{~L}$ - TAMP est évi-

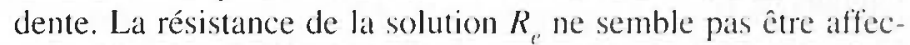
tée par la présence de l'albumine, comme on pouvait s'y altendre. Par contre, la présence de HSA augmente $C_{d}$, comme dans le cas de l'adsorption sur carbone vitreux $|17|$ el en accord avec des résultats antérieurs [19]. L'effet sur $R$, ne semble pas aussi important que dans une étude antérieure [19].

En ce qui concerne le film, l'albumine diminue fortement $R_{F}$, augmente $C_{F}$, mais ne semble pas affecter le coefficient de dispersion $\alpha_{F}$. Les désaccords observés par rapport aux résultats antérieurs proviennent sans doute du mode opératoire différent (immersion de l'EDT une heure en circuit ouvert avant mesure dans la référencel[9]).

En ce qui concerne la corrosion, nous avons montré par ailleurs que l'acier 316L est spontanément passivé et que la densité du courant de corrosion est inféricure à $1 \mu \mathrm{A} / \mathrm{cm} 2$ [19].

En effet, la vitesse de corrosion est déterminée par le courant de passivité el 
est peu sensible au changement de milieu agressif [191. L'effet du milieu, par comparaison avec une solution de $\mathrm{NaCl}$ seul (à 9 g/L), est essentiellement dû au ralentissement de la vitesse de réaction cathodique (déplacement de la branche cathodique d'environ $150 \mathrm{mV}$ dans TAMP). Ce phénomène est encore plus accentué par la présence d'albumine dans la solution. On constate donc l'effet inhibiteur du phosphate et de l'albumine sur le processus cathodique. Ces résultats sont en faveur d'une adsorption des ions phosphate et de l'albumine à la surface de l'électrode.

Par conséquent, si l'on se base sur les résultats obtenus à une date donnée après contact protéine-acier, on peut dire que la corrosion par piqûres est inhibée par le tampon phosphate et encore plus par l'albumine. Mais quelle est l'influence de HSA lorsque l'acier reste longtemps en contact avec le milieu?

\section{Comportement de l'interface en fonction du temps (EIS à différents temps)}

Il est évident que les conclusions relatives à ce comportement ne concerneront que les conditions opératoires (potentiel, vitesse de cisaillement, température, etc.) et l'intervalle de temps de contact considérés.

Les diagrammes d'impédance relevés à différents temps après immersion de l'EDT montrent (résultats non reportés ici) que les paramètres de la double couche (sauf $\alpha_{d}$ ) et du film présentent, à des degrés différents, des oscillations en fonction du temps, que ce soit en milieu TAMP ou HSA. De plus, l'albumine affecte tous les paramètres électriques relatifs aux deux boucles affaissées (valeur moyenne ou amplitude), en particulier $R_{\text {, et }} \alpha_{F}$ pour lesquels les oscillations semblent les plus importantes.

Autrement dit, l'influence de la protéine, quelle qu'elle soit, varie selon le temps de contact. Afin de discuter l'effet de HSA sur la corrosion, nous considérerons la variation temporelle de $R_{t}$, car inversement proportionnel au courant de corrosion, et celle de $\alpha_{l}$. car associé à l'homogénéité de la surface du film. La protéine agit en fait sur ce film, qui recouvre la surface de l'EDT au contact avec le milieu. Notons que plus $\alpha_{F}$ est grand plus la surface est homogène, la valeur maximale de $\alpha_{F}$ étant toujours de 1.

L'amplitude de la variation de $R$, pour HSA est bien plus importante que pour TAMP (fig. 4). Il est évident que les valcurs maximales de $R_{t}$ correspondent à une inhibition de la corrosion (courant de corrosion minimal) et vice versa. Au cours du temps, HSA inhibe ou favorise alternativement la corrosion. Ce comportement est effectivement dû à la protéine, car $R_{t}$ (TAMP) et $R_{t}$ (HSA) semblent être en opposition de phase. Malgré une incertitude expérimentale aux temps les plus longs, si la corrosion est inhibée dans HSA, elle semble favorisée dans TAMP.

Un comportement analogue s'observe pour $\alpha_{f}$ (fig. 5). L'homogénéité du film dans TAMP semble relativement constante, étant donné la faible variation de l'amplitude d'oscillation autour d'une valeur moyenne. En revanche, l'amplitude importante de $\alpha_{r}$. (entre 1 et $\approx 0,1$ ) dans le cas de HSA indique une variation très forte de l'influence de la protéine sur le film en fonction du temps. De plus, la valeur de $\alpha_{F}$ est proche de 1 à $t \cong 0$ dans les deux milieux, ce qui montre un film homogène au début de l'expérience. En présence de HSA, cependant, $\alpha_{r}$ diminue rapidement. entre environ 1000 et $3900 \mathrm{~s}$. Comme on sait par

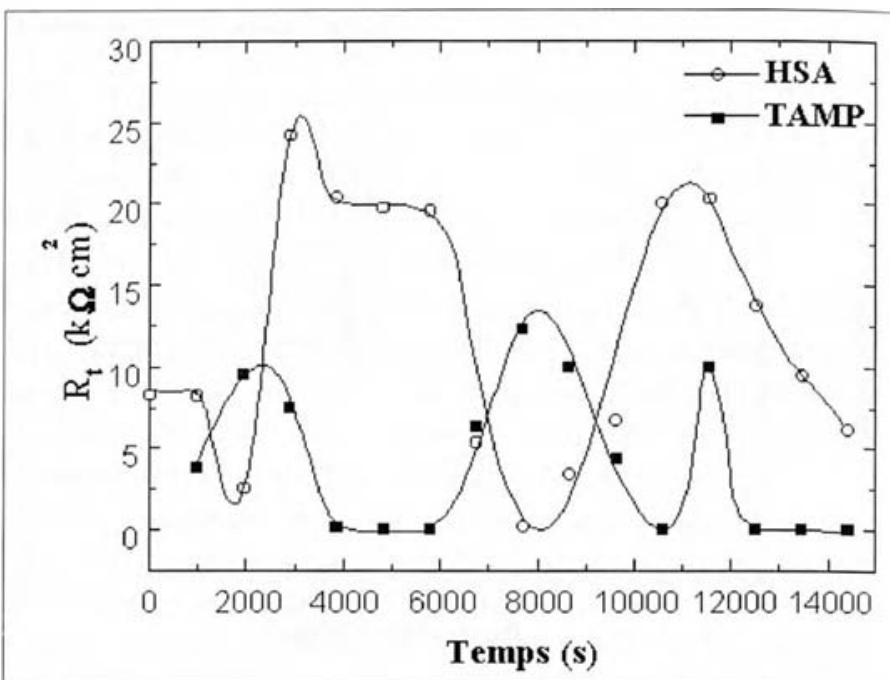

Fig. 4 - Évolution temporelle de la résistance de transfert de charge $I$ dans les deux milieux (mêmes conditions que pour les figures 1 et 2 ).

Fig. 4 - Time-evolution of the charge transfer resistance $R_{t}$ in both media (sam. conditions as in figures 1 and 2 ).

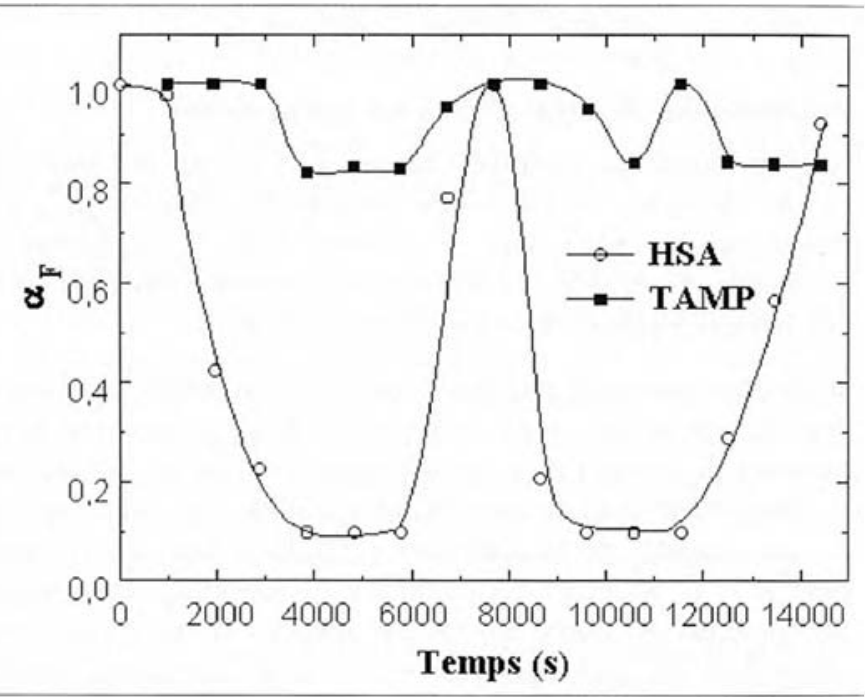

Fig. 5 - Évolution temporelle du coefficient de dispersion $\alpha_{F}$ du film sem conducteur dans les deux milieux (mêmes conditions que pour les fig. 1 et 2 Fig. 5 - Time evolution of the dispersion coefficient $\alpha_{F}$ of the semi-conductin film in both media (same conditions as in figares 1 and 2).

ailleurs que les protéines s'adsorbent aux interfaces hétéro gènes, on peut penser, dans le cas présent, que HSA s'adsorb de façon hétérogène, et ce jusqu'à un temps où $\alpha_{F}$ devien minimal $(\cong 0,1)$. Cependant, après ce premier temps critique $\alpha_{F}$ augmente rapidement, ce qui peut être expliqué par un désorption de HSA, jusqu'à ce que $\alpha_{F}$ redevienne maximal ( $\alpha$ $\cong 1$ ), comme à $t \cong 0$. Les processus d'adsorption et de désorp tion se déroulent alternativement à la surface du film condui sant au phénomène oscillatoire.

La figure 6 montre que $R_{t}$ et $\alpha_{F}$ varient en opposition de phas dans HSA, ce qui est en accord avec les conclusions tirées ci dessus. En effet, $R$, maximal correspond à $\alpha_{F}$ minimal, et don l'inhibition de la corrosion correspond bien à une adsorption d la protéine (et inversement).

Ainsi, la contradiction qui peut apparaître entre des conclusion: tirées de différentes mesures peut être due au fait qu'elles on été faites à des temps qui correspondent à des zones temporelles d'inhibition ou de non-inhibition. Ainsi, les mesures 


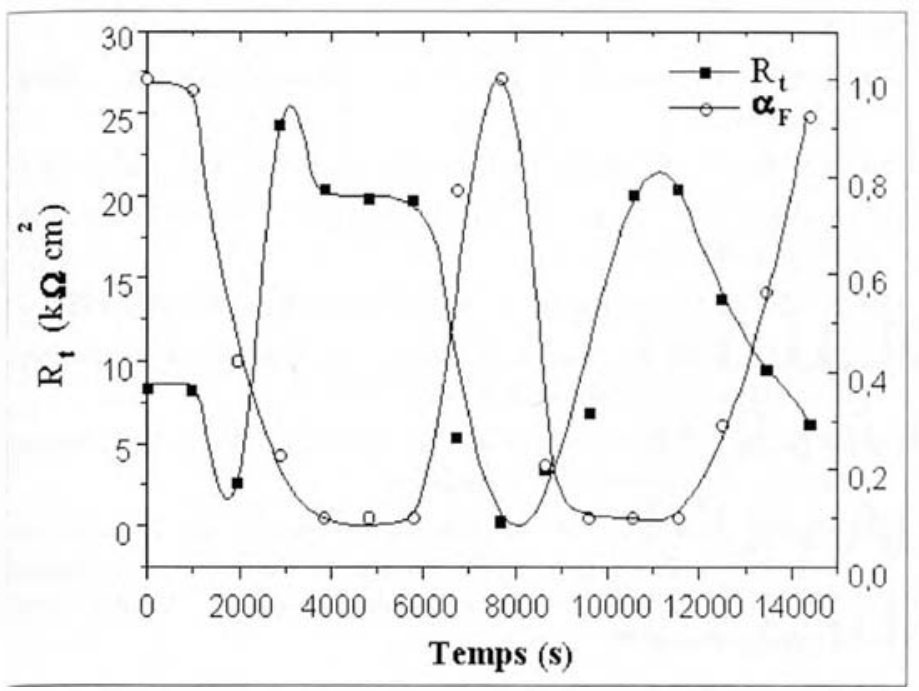

Fig. 6 - Variation en opposition de phase de la résistance de transfert de charge $R_{t}$ et du coefficient de dispersion $\alpha_{r}$ du film dans le milieu HSA.

Fig. 6 - Anti-phase shifi variation of the charge transfer resistance $R$, and the dispersion coefficient $\alpha_{F}$ of the film in the HSA medium.

effectuées ici juste après immersion indiqueraient un comportement semblable à celui du tampon, donc l'absence d'action de HSA, comme dans certains travaux de la littérature. Par contre, nos résultats antérieurs après $1 \mathrm{~h}$ d'immersion sont corroborés par la présente étude, car, à $3600 \mathrm{~s}$, le système se trouve dans une zone d'inhibition où $\alpha_{F}$ est quasi minimal et $R$, quasi maximal, compte tenu des incertitudes expérimentales (fig. 6).

Dans une publication antérieure, nous avons montré, sur la base de la variation d'une capacité interfaciale, que l'adsorption de HSA sur carbone vitreux, bien que non oscillatoire sur l'intervalle temps exploré $(<4000 \mathrm{~s}$ ), présente aussi deux constantes de temps. Par contre, un tel comportement oscillatoire a été révélé, pour des conditions identiques, dans le cas d'autres protéines plasmatiques humaines (fibrinogène, antithrombine III, globulines), les temps critiques paraissant dépendre de la vitesse de cisaillement. Ce comportement a été interprété, compte tenu de la structure et la charge de ces protéines au potentiel étudié, par l'alternance entre l'adsorption en multicouches et la désorption de ces molécules, suite à une synergie temps-écoulement agissant au-delà de la première couche [2I].

Dans le cas présent, le système est plus complexe du fait de la corrosion, et l'alternance adsorption-désorption peut être décrite par la séquence suivante :

1. Adsorption mono-moléculaire de HSA à la surface du film passif.

2. Adsorption de HSA au-delà de la couche mono-moléculaire conduisant à l'augmentation de $R_{\text {f }}$ (adsorption en multicouches).

3. Parallèlement, rigidification du film suite à l"adsorption ct distribution spattiale de l'effet protecteur, ce qui explique la diminution significative de $\alpha_{F}$ alor's que $R_{\text {l crô̂l. }}$

4. Désorption de HSA des couches supérieures sous l"iction de la contrainte de cisaillement. conduisant à l'augmentation rapide de $\alpha_{f}$ et la diminution de $R$.

5. Réapparition de la couche mono-moléculaire adtérée sur la surface du film attestée par les valeurs de $\alpha_{f}$ et $R_{t}$ proches de celles observées au début de l'expérience.

6. Reconstitution des couches supérieures (retour à l'étape 2).

Dans cette séquence, nous ne préjugeons pas du mode d'adsorption de la mono-couche, ni de la dénalturation (ou non) de la protéine dans cette première étape, celle-ci étant un concept généralement admis. Cependant, bien qu’une dénaturation partielle ait été montrée dans le cas de la $\beta$-lactoglobuline sur acier inoxydable en circuit ouvert, il est probable que la structure de HSA dans les couches supérieures est celle de la molécule native comme dans le cas la mono-couche de $\beta$-lactoglobuline adsorbée sur une surface hydrophile de $\mathrm{Si}: \mathrm{SiO}_{2}[22,23]$.

Du fait de sa structure (forme ellipsoüdale) el de la répartition de sa charge (selon trois domaines à l'état natif: $-9(1):-8(11)$ : $+2($ III)), qui lui confèrent un caractère dipolaire, HSA peut se fixer sur des sites occupés par des cations, des anions ou encore sur des sites nus du support. Elle peut "basculer", se mettre "couchée" et former ainsi des multicouches. En milieu électrolytique, cette protéine de transport fortement chargée (négativement à $\mathrm{pH}=7,4 \mathrm{vu}$ son point isoélectrique $\mathrm{pl} \cong 4,5$ ) peut fixer. par chélation, des ions, ce qui augmente sa chatge au cours du temps. Enfin, cette adsorption ayant lieu sur le film, sur lequel les ions phosphate jouent un rôle d'inhibiteur de corrosion (par rapport au milieu $\mathrm{NaCl}$ seul), les liens HSA-support sont plus labiles que sur le carbone vitreux (dans le domaine cathodique): ce qui explique que les molécules de HSA peuvent être plus facilement entraînées par l’écoulement. Enfin, en cé qui concerne la distribution spatiale de l'effet protecteur de HSA. elle peut être reliée à l'hétéroénergicité de l'adsorption. qui semble une propriété générale du comportement interfacial des protéines, l'adsorption initiale se faisant sur certains sites avec des constantes de vitesse maximales [24].

\section{Conclusion}

Cette étude sur le comportement temporel d'un acier inoxydable utilisé en chirurgie orthopédique, en tant qu'implants à court terme (ostéosynthèse) ou à long terme (prothèses osseuses), montre clairement que la durée du contact acier $316 \mathrm{~L}-$ milieu peut moduler l'influence d'une protéine sur la corrosion par piqûres.

L'existence de phénomènes oscillatoires, révélée ici comme dans le cas de l'adsorption sur carbone vitreux, par spectroscopie d'impédance électrochimique, montre que le caractère inhibiteur de corrosion d'une molécule peut varier en fonction du temps. Ainsi, une protéine peut avoir alternativement un effet d'inhibiteur de corrosion ou aucun effel (sans parler d'effer accélérateur) au cours du temps.

Enfin, ce comportement oscillatoire est probablement une propriété générale du comportement interfacial des protéines. du moins sous l'effet de l'écoulement.

\section{Remerciements}

M. EL OUAHABI remercie I'UNESCO pour l'attribution d'une bourse d'étude pour la réalisation de ce travail (Requête $\mathrm{N}^{\circ} 210$. 1). Nos plus vifs remerciements vont à R. BAvIERE et J. L. Hogivos (Société Solartron-France) pour leurs conseils et leur aide efficaces. 


\section{RÉFÉRENCES}

[1] M. A. Barbosa, Portugaliae Electrochim. Acta, 7, p.567, 1989.

12] J. D. Bryers, S. Hindricks, Ann. N. Y. Acad. Sci., 831, p. 127, 1997.

13] S.A. Brown, K. Merritt, J. Biomed. Mat. Res., 14, p. 173, 1980.

[4] S.A. Brown, K. MERrITr, Corrosion and Degradation of Implant Materials: Second Symposium ASTM STP 859, A. C. Farker and C. D. Griflin, Eds., American Society for Testing and Materials, Philadelphia, 1985, pp. 105-116.

15I M. Sivakumar, U K. Mudali, S. Rajeswari, J. Mat. Science, 28 , p. $6081,1993$.

[6] T. M. Leb, E. Cinang, J Mat. Science: Materials in Medicine, 9, p. $429,1998$.

17| S. R. SousA, M. A. Barbosı, J Mat. Sci. : Materials in Medicine, 2, p. 19, 1991.

[8] S. K. Chawla, S.A. Brown, K. Merritt, J. H. Payer, Comrosion, 46, p. 147, 1990 .

[9] Y. Nakayama, T. Yamamuro, Y. Kotoura, M. OKa, Biomaterials, 10, p. 420, 1989.

I10| V. J. Col.ancillo, N. D. Greene, D. B. Kettlekamp, H. Alexandi:R, C. J. CAMpliell, J. Biomed. Mater: Res., 1, p. 405, 1967.

[1I]S. STtintimanN, " Corrosion of surgical implants in vivo and in vitro tests ", Evaluation of Biomaterials, G. Winter, J. Leray, K. deGrott (eds), Wiley, Chichester, 1980, pp. 1-34.

[12] T. Nishizawa, T. Sasada, M. Morita, K. Mabuchi, The $33^{\text {ru }}$ Japan Congress on Materials Rescarch, March 1990, pp. 137-139.
[13] G. C. F. Clarck, D. F. Williams, J. Biomed. Mat. Res., 16, p $125,1982$.

[14] S.A. Brown, K. MerriTT, J. Biomed. Mat. Res., 15, p. 479, 1981.

[15] K. Merritt, S.A. Brown, N. A. Sharkey, J. Biomed. Mat. Res. 18, p. 1005, 1984.

[16] K. Meirritt, S.A. Brown, J. Biomed. Mat. Res., 22, 11 ], 1988.

[17] M. Marie de Ficquelmont-Lolzos, W Kanté, H Takenouti J. Electroanal. Chem., 428, p. 129, 1997.

[18] W. Kanté, Thèse de Doctorat de l’Université Paris XIII, Institu Galilée, Villetaneuse, 19 mars 2001.

[19] M. El Ouahabi, M. Marie de Ficquelmont-Loizos, H Takenouti, P. Lambin, F. El Mdari, S. El Adioul, A. Srhiri $13^{\text {tmm }}$ Forum sur les Impédances Électrochimiques, CNRS, Paris 11 décembre 2000.

[20] S. Omanovic, S. G. Roscoe, Langmuir, 15, p. 8315, 1999

[21] W. Kanté, H Takenouti, M. Marie de Ficquelmont-Loizos $14^{\text {tmi }}$ Forum sur des Impédances Électrochimiques, CNRS, Paris 14 janvier 2002.

[22] S. Omanovic, S. G. Roscoe, J. Colloid Interf. Sci, 227, p. 452 2000.

[23] R. J. Marsh, R. A. L. Jones, M. Sferrazza, J. Penfold, ibidem 218, 347, 1999 .

[24] A. N. Asanov, L. J. Delucas, P. B. Oldham, W.W. Wilson, I Coll. Interf. Sci., 191, p. 222, 1997.

\section{MATÉRIAUX \%ั TECHNIQUES}

Intelligent materials and fluid systems,

J. TANI (Tohoku University, Japan)

Role of the Prandt number on the control of an unstable flow by

action on the wall temperature.

C. Bénard, J. Liu, H. Pabiou (Paris)

Effective laminar flow control by selective suction system on swept wing flow.

Y. EGAMI, Y. KoHama (Tohoku University, Sendai)

Homogeneisation methods applied to model the thermomechanical behavior of shape memory alloys,

E. PATOOR and M. Berveiller (ENSAM Metz)

Sudy of vibration control system of CFRP composite beam with piecoceramics and electro-rheological fluids actuators,

T. TAKAWA (Setsunan Univ), T. FukUdA (Osaka City Univ.)

Durability of structures and smart materials,

P.-F. Gobin (INSA, Lyon), M. SALviA (ECL), M. Morin et Y. JAYET (INSA. Lyon)

\section{Intelligent materials and structures}

conferences selected from the
The piezoelectric implant: its application to the in situ monitoring of materials,

Y. JAYET, J.-C. BABOUX (INSA, Lyon)

In situ cure monitoring of composite materials in autoclave molding, T. FUKUDA (Osaka City University)

Future trends in health monitoring of materials, J.-Y. CAVAILLÉ (INSA Lyon)

Identification of delamination crack of graphitelepoxy laminates by electric potential method for health monitoring,

A. TODOROKI and H. Suzuki (Tokyo Institute of Technology)

Ferro-Fluids : nanomotors and namogenerators,

F. Gazeau, C. Baravian, J.C. Bacri, R. Perzynski, M.I. Shliomis (Univ. Paris 6/Weizmann Institute of Science, Israel)

Misting free E.B. treatment for endoscope,

K. Oguri, N. Iwataka, H. Izumi, A. Tonegawa, K. Takayama, Y. NISHI (Tokai University) 